T cell response could be localised to a few amino acids around the tenth residue of the $\mathrm{B}$ chain. On the other hand the specificity of antibodies produced by these animals did not reflect the specific determinants unique to the insulin used for immunisation. This distinction between $\mathrm{T}$ cell response determinants and specificity at the serum antibody or B cell level was also shown in mice.

\title{
Nuclear quadrupole resonance at Toulouse
}

from J.A.S. Smith

P. GrIvit (Universite de Paris), in whose laboratory the first nuclear quadrupole resonance experiments in France were conducted, opened the conference*. He reviewed in masterly fashion the many current applications at Orsay of nuclear quadrupole interactions to solid state physics, concluding with the description of a sensitive pulsed spectrometer designed by L. Guibé and his coworkers (Paris, Orsay), a feature of which was its highly automated operation. This theme was repeated in several other papers, notably from E.A.C. Lucken's group (University of Geneva) who described a pulsed FT spectrometer for the study of single crystals, from which the complete electric field gradient tensor may be derived under computer control. Other workers described frequency and phase-locked spectrometers for the determination of true line shapes (P.T. Narasimhan, Indian Institute of Technology, Kanpur) and a special pulsed coil system for detecting ${ }^{14} \mathrm{~N}$ signals from samples external to the spectrometer ( $T$. Hirschfeld \& S.M. Klainer, University of California, Berkeley) with potential applications to geochemistry. A recent development, the derivation of nuclear quadrupole interactions in free radicals by ENDOR experiments, was described by A. Colligiani (CNR Quantum Chemistry Laboratory, Pisa), who reported the first measurement of the full ${ }^{2} \mathrm{H}$ quadrupole coupling tensor in the free radical HOOC.CD.COO ${ }^{-}$.

The applications of nuclear quadrupole resonance to transition metal chemistry were emphasised. T.B Brill (University of Delaware,) described the applications of ${ }^{55} \mathrm{Mn}$ quadrupole resonance to the study of the electronic structure of tricarbonylcyclohexadienylmanganese(I) and its substituted analogues, and other speakers discussed ${ }^{63} \mathrm{Cu}$ resonance in diamagnetic copper(II) complexes (T. Okuda \& A. Weiss, Technical High School, Darmstadt), ${ }^{35} \mathrm{Cl}$ quadrupole resonance in mercury pentachlorocyclopentadienes (G. Wulfsberg and coworkers, St John's University, Collegeville, Minnesota), in some hexachloroplatinates (IV) hexahydrates (A. Sasane, Shinshu University, Japan), and

-The fifth international symposium on Nuclear Quadrupole Resonance Spectroscopy was held in Toulouse, France on 10-14 $P a r i s)$ and $G$. Jugie (CNR, Toulouse).
Zeeman studies of ${ }^{63} \mathrm{Cu}$ resonance in single crystals of $\mathrm{Cu}(\mathrm{I})$ triphenylphosphine dimers (H. Negita et al. Hiroshima University, Japan). Although it has been known for some time that quadrupole resonance of ligand nuclei can be readily studied in paramagnetic complexes, for example ${ }^{14} \mathrm{~N}$ quadrupole resonance in dichloro (dimethylnitrosoamine) copper (II) (D. Nakamura, Nagoya University, Japan), T.J. Bastow and H.J. Whitfield (CSIRO, Clayton, Australia) showed in their paper that strong ${ }^{63} \mathrm{Cu}$ signals can be detected in several paramagnetic $\mathrm{Cu}(\mathrm{II})$ salts such as $\mathrm{CuCl}_{2}$ and $\mathrm{CuBr}_{2}$; the signals are seen because of strong exchange narrowing, with widths of about $35 \mathrm{kHz}$, little different from that of ${ }^{63} \mathrm{Cu}$ resonance in diamagnetic $\mathrm{Cu}_{2} \mathrm{O}$. In a review of recent studies of inorganic coordination compounds, T.L. Brown (University of Illinois) discussed the wealth of information now forthcoming from solid complexes by a combination of conventional cw and pulsed measurements with double resonance techniques.

The remarkable increase in sensitivity afforded by double resonance experiments was emphasised by many speakers. For ${ }^{2} \mathrm{H}$ and ${ }^{14} \mathrm{~N}$, the technique is now well established; J.L. Ragle (University of Massachusetts, Amherst) demonstrated the usefulness of ${ }^{2} \mathrm{H}$ quadrupole resonance in a study of pyridine and its salts, and ${ }^{14} \mathrm{~N}$ double resonance was applied by $M$. Garcia (Queen Elizabeth College, London) to many imidazole derivatives and by $\mathrm{A}$. Loetz and $J$. Voitländer (University of Munich) to a series of amidinium salts. Three papers were devoted to another recent development of double resonance spectroscopy, that of the detection of ${ }^{17} \mathrm{O}$ quadrupole resonance signals in natural abundance; J.A.S. Smith (Queen Elizabeth College, London) and S.G.P. Brosnan and D.T. Edmonds (University of Oxford) discussed the novel features of such methods applied to compounds containing -O-H groups, the most important of which is the appearance of dipolar structure, and $O$. Lumpkin (University of California, San Diego) described its applications to compounds containing $\mathrm{O}-\mathrm{O}$ bonds, such as $\mathrm{IrO}_{2} \mathrm{Cl}(\mathrm{CO})\left(\mathrm{PO}_{3}\right)_{2}$ a reversible oxygen carrier. In a later paper, P.J. Bray (Brown University, Rhode Island) demonstrated very clearly the significance of ${ }^{14} \mathrm{~N}$ quadrupole coupling constants as a measure of biological activity, for example in the bateriostatic activity of the sulphanilamides and the tumour-inhibiting properties of several nitrogen mustards.

Studies of phase transitions and molecular motion in solids were common themes of a number of papers. J.R. Brookeman (University of Florida) gave a timely review of the importance of measurements of the resonance frequency and relaxation times close to phase transitions and several other papers discussed the temperature dependence of the same quantities for example, in hydrazine derivatives (Y. Abe, University of Tsukuba, Japan), in some bromocyclotriphosphazatrienes (A.L. Porte, University of Glasgow) and in ammonium salts such as $\alpha-\mathrm{NH}_{4} \mathrm{HgCl}_{3}$ and $\mathrm{NH}_{4} \mathrm{ReO}_{4}$ (H. Chihara, Osaka University, Japan) in which the anomalous temperature coefficients were interpreted by taking into account the finite jump-time of the $\mathrm{NH}_{4}{ }^{+}$ ion between its equilibrium configurations. There was increasing interest in the value of variable pressure studies in studying solidstate processes; R.J.C. Brown (Queen's University, Kingston, Canada) stressed the generality of the relationship between the temperature and pressure coefficients of the quadrupole resonance frequency and $J$. Stankowski (Poznań, Poland) studied ${ }^{75} \mathrm{As}$ resonance in $\mathrm{K} \mathrm{D}_{2} \mathrm{AsO}_{4}$ up to $3 \mathrm{kbar}$ as a test of current theories of proton-ordering in this crystal. I.G. Shaposhnikov (Perm, USSR) discussed a new theoretical attempt to analyse the 'Bayer' problem, the temperature variation of the quadrupole spin-lattice relaxation time, $T_{1 \mathrm{Q}}$, due to random thermal oscillations, by the methods of quantum kinetics; his theory shows that significant departures of $T_{\mathrm{lQ}}$ from the predictions of the Bayer equations can be expected at temperatures above $T_{m / 5}, T_{m}$ being the temperature at which orientational order disappears. Experimental aspects of relaxation time measurements were referred to by A.H. Brunetti (IMAF, Cordoba, Argentina) and there was some discussion of the usefulness of $T_{2}^{*}$, the spin-phase memory time, by S. Hacobian (University of Sydney) and M.A. Whitehead and coworker (McGill University).

Nuclear quadrupole resonance spectroscopists have long been worried about high-order nuclear electrical multipole contributions to their measured frequencies; S.L. Segel (Queen's University, Kingston) showed by careful frequency measurements on a number of nuclei such as ${ }^{185.187} \mathrm{Re}$ in $\mathrm{NH}_{4} \mathrm{ReO}_{4},{ }^{209} \mathrm{Bi}$ in $\mathrm{BiCl}_{3}$ and ${ }^{93} \mathrm{Nb}$ in $\mathrm{NbCl}_{5}$ that there were no measurable hexadecapole contributions. The question as to whether this is generally true still remains.

J.A.S. Smith is Professor in the Department of Chemistry, Queen Elizabeth College, London. 